\title{
Vancomycin blocks autophagy and induces interleukin-1及 release in macrophages
}

\author{
Young Eun $\mathrm{Ha}^{1}$, Kyoung-Hye Kong ${ }^{2,3}$, Mi-Hyang $\mathrm{Cho}^{2,3}$, Dong-Hou Kim²,3, Young-Sup Song ${ }^{3,4}$ \\ and Seung-Yong Yoon ${ }^{2,3}$
}

\begin{abstract}
Systemic inflammatory response syndrome (SIRS) is a serious condition that can cause organ failure as an exaggerated immunoresponse to the infection or other causes. Recently, autophagy was reported as a key process that regulates inflammatory responses in macrophages. Vancomycin is one of the most commonly prescribed antibiotics for sepsis treatment or following surgery. However, there are no studies on how vancomycin affects autophagy or inflammation. Here, we treated macrophage cell lines with vancomycin and lipopolysaccharides and found that vancomycin blocks autophagy and increases inflammatory responses. This finding suggests that vancomycin should be more cautiously administered in order to prevent unwanted SIRS during sepsis.
\end{abstract}

The Journal of Antibiotics (2015) 68, 76-80; doi:10.1038/ja.2014.112; published online 20 August 2014

\section{INTRODUCTION}

Systemic inflammatory response syndrome is a serious condition that can result in organ failure and death as an exaggerated immune response to the infection or other causes. Hence, inflammatory control is a very important issue when managing sepsis.

Autophagy is the process of sequestering cytoplasm, including excess or aberrant organelles and misfolded proteins, into doublemembrane vesicles for delivery to degradative organelles (that is, the lysosome) for the breakdown and eventual recycling of the resulting macromolecule. ${ }^{1}$ Interestingly, autophagy was recently recognized as important to the regulation of innate immune responses. ${ }^{2-4}$ Depletion of autophagic proteins promotes mitochondrial dysfunction and interleukin (IL)-1 $\beta$ and -18 secretion in response to the presence of lipopolysaccharide (LPS) and ATP in macrophages. ${ }^{5}$ Autophagydeficient mice demonstrate more inflammation in sepsis models and are susceptible to LPS-induced mortality, ${ }^{5}$ thus suggesting their importance in regulating autophagy-related systemic inflammation in macrophages.

Vancomycin is one of most commonly prescribed antibiotics for the treatment of sepsis or following surgery because it targets Gram-positive bacteria such as methicillin-resistant Staphylococcus aureus. However, there are no studies on how vancomycin affects autophagy and inflammation. Here, we report for the first time that vancomycin blocks autophagy and induces inflammatory responses in macrophages. This finding suggests that vancomycin should be more cautiously administered in order to prevent unwanted systemic inflammatory response syndrome during sepsis.

\section{MATERIALS AND METHODS}

\section{Cell culture}

The THP-1 human monocytic leukemia cell line and Raw264.7 mouse macrophage-like cell line were cultured in 100-mm dishes (Nunc, Penfield, NY, USA) in culture medium (Dulbecco's modified Eagle's medium/high glucose (Invitrogen, Carlsbad, CA, USA) with 5\% fetal bovine serum (Invitrogen) and 1\% PenStrep (Invitrogen)). The cells were subcultured to $70-80 \%$ confluency using HBSS.

\section{Drug treatment}

Vancomycin (Boehringer Mannheim, Basel, Switzerland) was added to the cells at the final concentrations indicated. For the control experiments, $0.001 \%$ dimethyl sulfoxide was administered.

\section{Western blot analysis}

For western blot analysis, cultures were scraped using a cell scraper and centrifuged at 2000 r.p.m. for $5 \mathrm{~min}$. Cell pellets were resuspended in protein extraction solution (Pro-Prep; Intron, SungNam, Korea) according to the manufacturer's guidelines and incubated at $-20^{\circ} \mathrm{C}$ for $20 \mathrm{~min}$. The suspension was centrifuged at 13000 r.p.m. $\left(4^{\circ} \mathrm{C}\right)$ for $5 \mathrm{~min}$, and the supernatant was transferred to $1.5-\mathrm{ml}$ tubes. Protein concentrations were measured using the Bradford method. Equal amounts of protein were mixed with sample buffer (62.5 mm Tris- $\mathrm{HCl}$ (pH 6.8), 1\% SDS, 2.5\% glycerol, 0.5\% 2- $\beta$-mercaptoethanol, and bromophenol blue), boiled at $100{ }^{\circ} \mathrm{C}$ for $5 \mathrm{~min}$, and stored at $-20^{\circ} \mathrm{C}$ until use. Proteins were subjected to SDS-polyacrylamide gel electrophoresis at a constant voltage $(130 \mathrm{~V})$, transferred to polyvinylidene difluoride membranes (0.2-mm pores; Bio-Rad Laboratories, Hercules, CA, USA), and further maintained at $110 \mathrm{~V}$ for $2 \mathrm{~h}$. After 1-h incubation and blocking in Tris-Buffered Saline and Tween 20 (TBST) buffer (10 mm Tris, $100 \mathrm{~mm} \mathrm{NaCl,}$ $0.1 \%$ Tween-20) containing $2 \%$ bovine serum albumin and $2 \%$ normal horse

${ }^{1}$ Samsung Medical Center, Department of Infectious Diseases, Sungkyunkwan University School of Medicine, Seoul, Korea; ${ }^{2}$ Department of Anatomy and Cell Biology, University of Ulsan College of Medicine, Seoul, Korea; ${ }^{3}$ Cell Dysfunction Research Center (CDRC), Bio-Medical Institute of Technology (BMIT), University of Ulsan College of Medicine, Seoul, Korea and ${ }^{4}$ Department of Biomedical Sciences, University of Ulsan College of Medicine, Seoul, Korea

Correspondence: Professor S-Y Yoon, Department of Anatomy and Cell Biology, University of Ulsan College of Medicine, 88, Olympic-ro 43-gil, SongPa-Gu, Seoul 138-736, Korea.

E-mail: ysy@amc.seoul.kr

Received 20 February 2014; revised 23 June 2014; accepted 27 June 2014; published online 20 August 2014 
serum, the blots were treated with antibodies for $16 \mathrm{~h}$ at $4{ }^{\circ} \mathrm{C}(1: 1000$ rabbit LC3 (Sigma-Aldrich, St Louis, MO, USA), 1:1000 rabbit p62/SQSTM1 (Cell Signaling Technology, Beverly, MA, USA), and 1:1000 mouse $\beta$-actin (Sigma-Aldrich)). Blots were washed in TBST buffer, incubated with horseradish peroxidase-labeled anti-IgG (1:5000; Pierce, Rockford, IL, USA), and visualized using enhanced chemiluminescence reagent (Amersham, Arlington Heights, IL, USA) and X-ray film.

\section{Immunocytochemistry}

Cells were grown on glass coverslips and fixed in $4 \%$ paraformaldehyde in $0.1 \mathrm{M}$ phosphate buffer for $30 \mathrm{~min}$ at room temperature, and cell membranes were permeabilized by incubation for $30 \mathrm{~min}$ in $0.05 \mathrm{M}$ Tris buffer ( $\mathrm{pH} 7.4$ ) containing $0.1 \%$ Triton X-100, $2 \%$ bovine serum albumin, and $2 \%$ normal horse serum. Cultures were incubated overnight at $4{ }^{\circ} \mathrm{C}$ in primary antibody solution (1:1000 rabbit LC3; Sigma-Aldrich), washed, and incubated with fluorescein isothiocyanate (FITC)- or Cy3-labeled secondary antiserum (1:300; Jackson Laboratories, West Grove, PA, USA). Images were obtained using a DMIRB microscope (Leica, Wetzlar, Germany) and CoolSNAP-cf CCD-camera (Roper Scientific, Sarasota, FL, USA). For double immunostaining, two
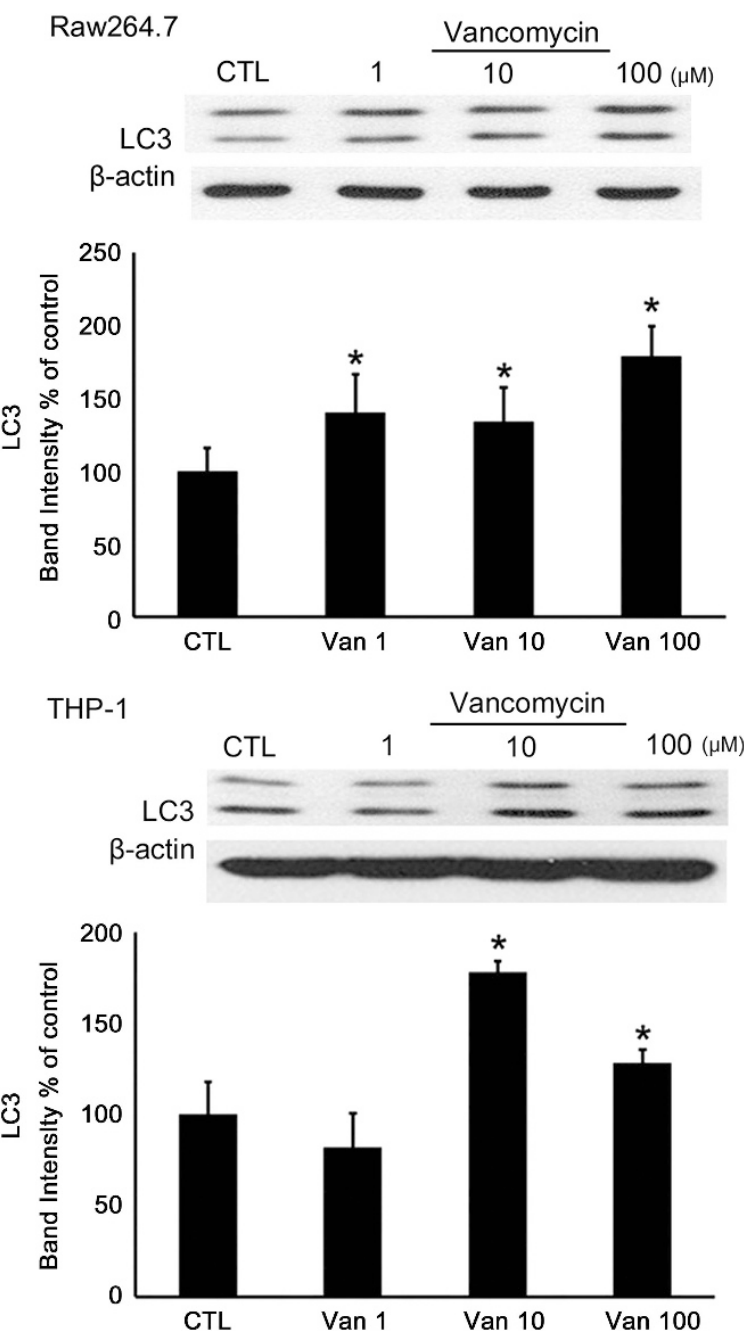

Figure 1 LC3-II increase in vancomycin-treated macrophage cell lines. Representative western blots of control and vancomycin-treated Raw264.7 and THP-1 cells. The band intensity shows the LC3-II increase in vancomycin-treated cells in comparison to controls (CTL). LC3-II increased compared to control. Data are the mean \pm s.d. of three independent experiments ( $n>200$ independent neurons) and were analyzed using the Student's $t$-test. ${ }^{*} P<0.05$ vs CTL. antibodies from different species were selected and a band-pass fluorescence filter was used. Negative control stainings (from which the primary antibody was omitted) were almost blank.

\section{DNA transfection}

Cultures were transfected with mCherry-GFP-LC3 plasmids using a Lipofectamine 2000 mixture (Invitrogen). mCherry-GFP-LC3 plasmids were diluted in Opti-MEM medium (Invitrogen) to a final concentration of $800 \mathrm{ng}$ per $50 \mu \mathrm{l}$, and Lipofectamine 2000 was diluted in Opti-MEM to a final concentration of $2 \mu$ per $50 \mu$ l. The diluted plasmids were added to the diluted Lipofectamine 2000 to obtain a ratio of $2 \mu$ l Lipofectamine 2000 per $0.8 \mu \mathrm{g}$ plasmid, and then the mixture was added to the cultures to obtain a final concentration of $800 \mathrm{n}$ per well. After 1.5 or $4 \mathrm{~h}$ of incubation, the cell media were replaced with fresh prewarmed media.

\section{Enzyme-linked immunosorbent assay}

The cell culture supernatants were assayed for IL-1 $\beta$ using ELISA kits (R\&D Systems, Minneapolis, MN, USA) according to the manufacturer's

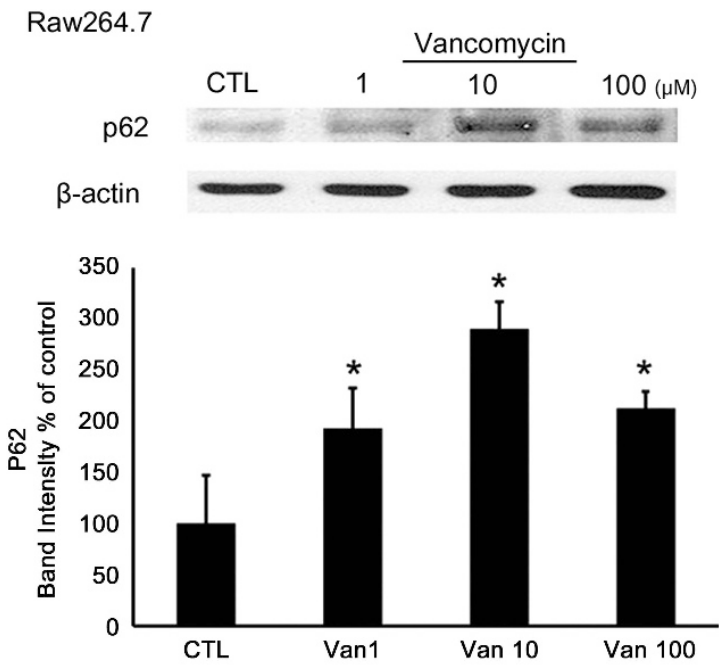

THP-1

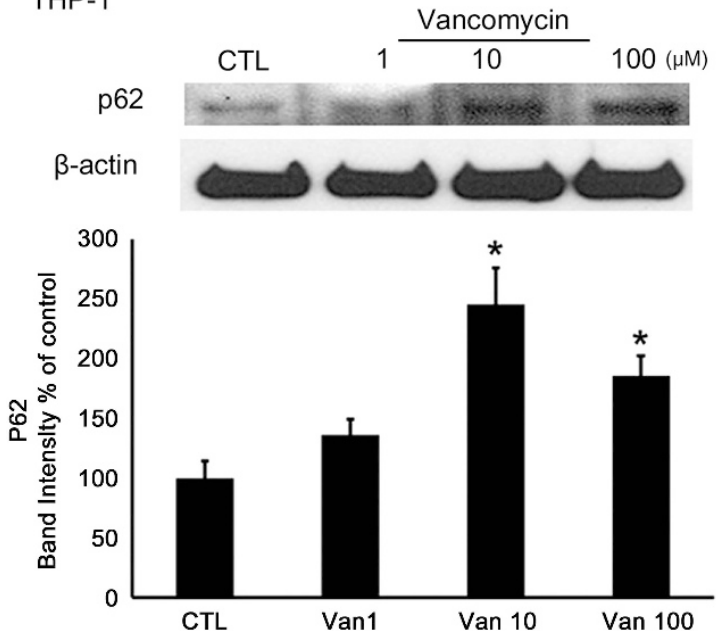

Figure 2 Increase inp62/SQSTM1 in vancomycin-treated macrophage cell lines. Representative western blots of control and vancomycin-treated Raw264.7 and THP-1 cells. The band intensity of p62/SQSTM1 (sequestosome-1) is greater in vancomycin-treated cells in comparison to the controls (CTL). p62/SQSTM1 increased compared with control. Data are the mean \pm s.d. of three independent experiments $(n>200$ independent neurons) and were analyzed using the Student's $t$-test. ${ }^{*} P<0.05$ vs CTL. 
instructions. Briefly, the supernatants and recombinant mouse IL-1 $\beta$ (used as the standard) were combined with mouse IL-1 $\beta$ capture antibody $\left(4 \mu \mathrm{g} \mathrm{ml}^{-1}\right)$ coated 96-well enzyme-linked immunosorbent assay plates. After $2 \mathrm{~h}$ of incubation at room temperature, the plate was washed $3 \times$ with wash buffer $(0.05 \%$ Tween-20 in phosphate-buffered saline ( $\mathrm{pH} 7.4)$ ), and then mouse IL-1 $\beta$ biotinylated detection antibody solutions $\left(0.4 \mu \mathrm{g} \mathrm{ml}^{-1}\right)$ were added to the plate. After $2 \mathrm{~h}$ of incubation at room temperature, the plate was washed $3 \times$ with wash buffer $(0.05 \%$ Tween- 20 in phosphate-buffered saline ( $\mathrm{pH}$ 7.4)), streptavidin-horseradish peroxidase was added to each well, the plate was then incubated for $20 \mathrm{~min}$ at room temperature, and antigens were finally visualized using a substrate solution in the dark and a microtiter plate reader set to $450 \mathrm{~nm}$.

\section{Transfection of small interfering RNA}

The Raw264.7 cells were transfected with scrambled small interfering RNA (siCTL) or siAtg7 using Lipofectamine 2000 (Invitrogen, \#11668-019) as per the manufacturer's guide

\section{Statistical analysis}

Data were analyzed using the Student's $t$-test and are presented as the mean \pm s.d. In this study, $P<0.05$ is considered statistically significant.

\section{RESULTS}

Increase in autophagosomes in vancomycin-treated macrophage cell lines

To determine whether vancomycin affects autophagy in macrophages, western blotting analysis was performed on the THP-1 and Raw264.7 cell lines. These cell lines were treated with 1,10 or $100 \mu \mathrm{M}$ vancomycin for $24 \mathrm{~h}$. LC3-II, a lipidated LC3 that is a marker of autophagic vacuoles, increased in the vancomycin-treated macrophage cell lines compared with control (Figure 1), thereby indicating the vancomycin-dependent increase in autophagosomes in the macrophages.

Blockage of autophagic flow in vancomycin-treated macrophage cell lines

Two mechanisms are responsible for increasing LC3-II. First, an increase in autophagic influx may increase LC3-II. Second, impairment of the autophagolysosome fusion process may result inLC3-II accumulation. ${ }^{6}$ To determine whether vancomycin enhances or blocks autophagosomal flux, we first assessed p62/sequestosome-1 (SQSTM1), a well-known autophagic substrate. p62/SQSTM1 increased in the vancomycin-treated macrophages compared with

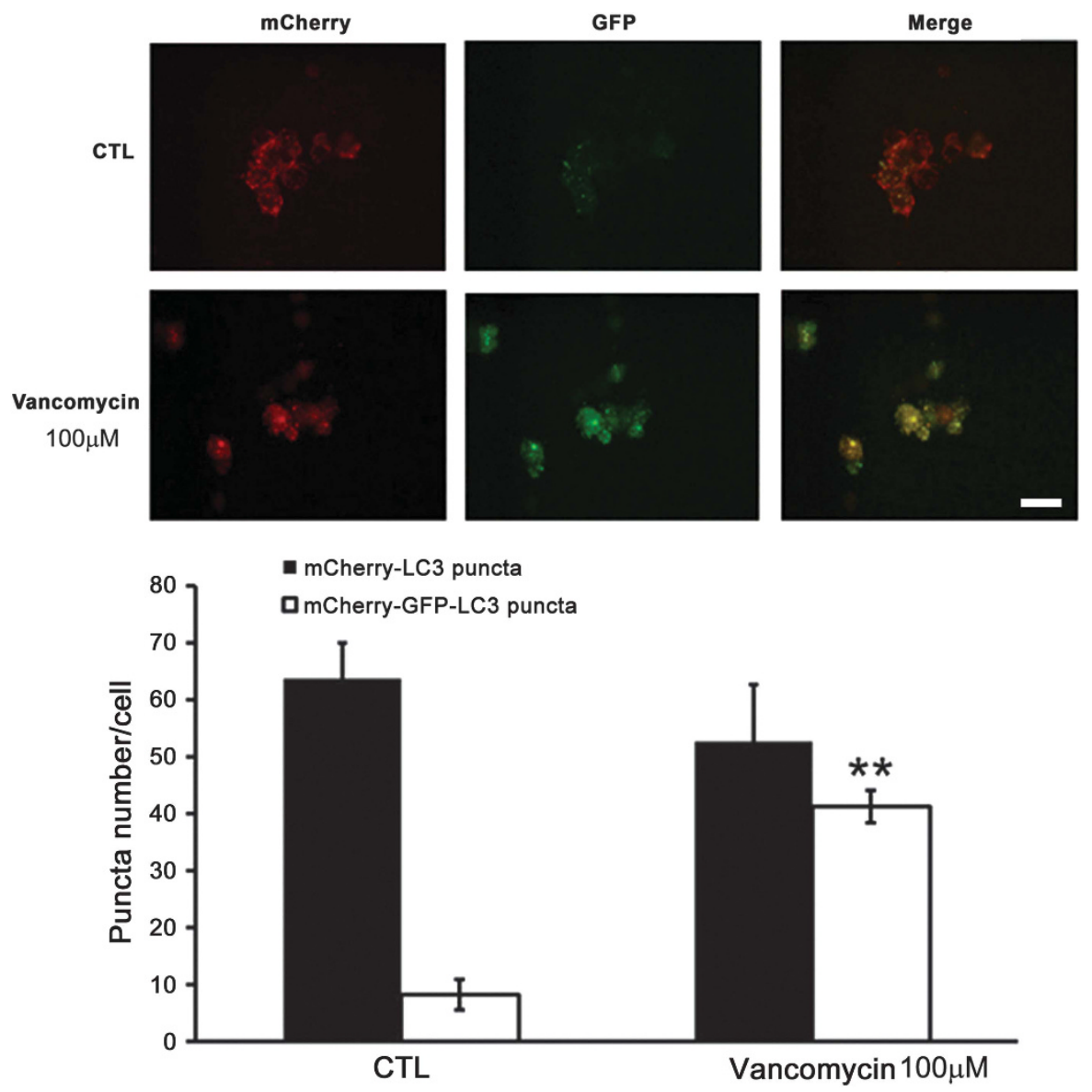

Figure 3 Blocking autophagic flux in Raw264.7 cells using vancomycin. Representative immunofluorescence images of vancomycin-treated Raw 264.7 and control (CTL) cells that were transfected with mCherry-GFP-LC3. Note the increase in LC3-GFP dots in $100 \mu \mathrm{m}$ vancomycin-treated cells. The merged signals increased after vancomycin treatment, suggesting that vancomycin blocks autophagic flux. The lower graph compares the puncta numbers of mCherry-LC3 (black bars) and mCherry-GFP-LC3 (white bars) between $100 \mu \mathrm{m}$ vancomycin-treated Raw264.7 and CTL cells. $100 \mu \mathrm{m}$ Vancomycin-treated cells had increased puncta and colocalization. Data are the mean \pm s.d. of three independent experiments $(n>200$ independent neurons) and were analyzed using the Student's $t$-test. Scale bar $=20 \mu \mathrm{m},{ }^{* *} P<0.01$ vs CTL. 
control (Figure 2), indicating the vancomycin-dependent blockade of autophagy flux in macrophages. We also transfected the macrophage cell lines with a tandem repeat GFP-mCherry-LC3 construct. In this system, the GFP (green fluorescent protein) signal is sensitive to the acidic and/or proteolytic conditions of the lysosome lumen, whereas mCherry is more stable. ${ }^{7}$ Therefore, the colocalization of GFP and mCherry fluorescence indicates that the compartment did not fuse with the lysosome (for example, a phagophore or autophagosome). In contrast, mCherry signaling without GFP corresponds to an amphisome or autolysosome. ${ }^{8}$ Treatment with vancomycin increased both GFP-LC3 and mCherry-LC3 fluorescent puncta and their apparent colocalization (Figure 3). By calculating the proportion of merged signals to all signals, we found that the proportion of merged (yellow) signals increased in vancomycintreated Raw264.7 cells, suggesting that vancomycin blocks autophagic flux (Figure 3).

\section{Increase in inflammatory mediators in vancomycin-treated} macrophage cell lines

As it was recently reported that autophagy regulates inflammatory responses in macrophages and is aggravated by autophagy dysfunction, ${ }^{5}$ we speculated that vancomycin-induced autophagy dysfunction may aggravate the inflammatory response of macrophages. Hence, the THP-1 and Raw 264.7 cells were treated with LPS plus vancomycin for $24 \mathrm{~h}$ to determine whether vancomycin aggravates LPS-induced

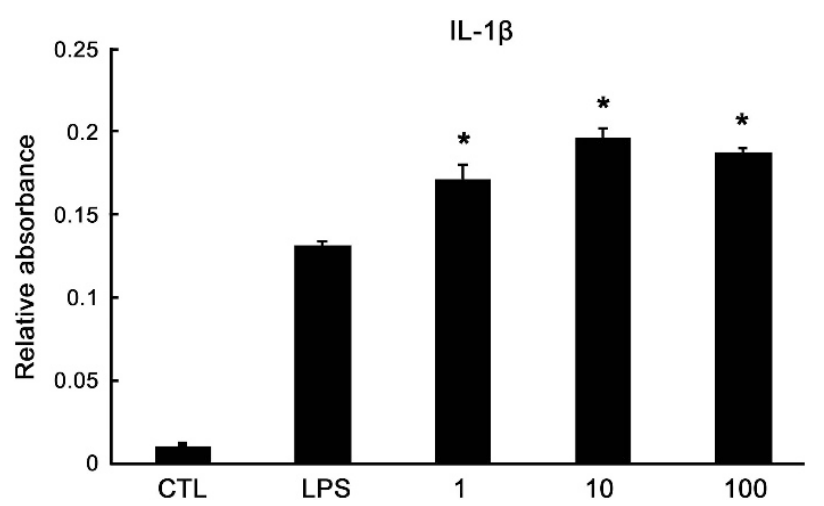

Figure 4 Interleukin-1 (IL-1) aggravation. Representative graphs of enzymelinked immunosorbent assay data showing $\mathrm{IL}-1 \beta$ in vancomycin-treated Raw264.7and CTL cells. IL-1 $\beta$ release is higher in vancomycin-pluslipopolysaccharide (LPS)-treated cells in comparison to LPS-only-treated cells. ${ }^{*} P<0.05$ vs $\mathrm{CTL}$. inflammation. IL-1 $\beta$ release into the media (as measured by enzymelinked immunosorbent assay) demonstrated a greater increase in macrophages when LPS and vancomycin were added in comparison with only LPS (Figure 4), further suggesting inflammatory aggravation after the impairment of autophagy.

To identify the relationships between autophagy and IL-1 $\beta$ release in vancomycin-treated cells, we transfected the cells with siAtg7, a key molecule to induce autophagy. Interestingly, we found that Atg7 knock down decreased the release of IL-1 $\beta$ in vancomycin-treated cells (Figure 5b), suggesting that reduction of autophagy induction by Atg7 knockdown could diminish autophagic blockade and decrease IL-1 $\beta$ release, because vancomycin blocked the late phase of autophagy like lysosomal fusions which may aggravate IL-1 $\beta$ release.

\section{DISCUSSION}

We report for the first time that vancomycin blocks autophagy in macrophages and increases the inflammatory response. Although main function of antibiotics is related to eliminate microorganisms, their distinct effects on inflammation or microcirculation have become understood. ${ }^{9}$ Our current study will shed new light on the understandings of this field. Our findings suggest that vancomycin should be administered more cautiously in order to prevent unwanted SIRS in patients suffering from sepsis or other conditions.

One way to avoid the proinflammatory effects of vancomycin is to thoroughly monitor patient symptoms, signs, and laboratory data (for example, IL-1 $\beta$ ). If there is an increase in serum IL- $1 \beta$, we should consider altering the vancomycin treatment regimen (for example, replacement with other antibiotics) or administering vancomycin with anti-inflammatory or autophagy-inducing drugs such as rapamycin. In addition, because autophagy plays a role in bacterial phagocytosis, ${ }^{2-4}$ our present results also suggest that vancomycin might interfere with bacterial lysis by macrophages. Because $S$. aureus subverts autophagy for replication and host cell death, ${ }^{10}$ our findings may suggest that vancomycin might interfere with $S$. aureus-induced replication and host cell death. These aspects require further testing and validation.

Our current findings may be applicable to clinical research. The mechanism of vancomycin toxicity, like nephrotoxicity, has not been clarified to date. ${ }^{11}$ Nephrotoxicity is not rare, so it is important to develop a method that prevents it. The autophagy-blocking effects of vancomycin might have a role in nephrotoxicity. This should be tested in the future in order to develop new ways to administer vancomycin. Moreover, as super-bacteria that are resistant to traditional antibiotics continue to emerge, vancomycin has become the only choice for treating super-bacteria-infected patients. Restricting the use of a
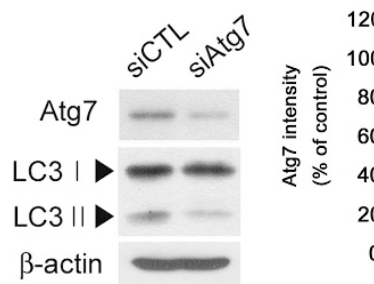
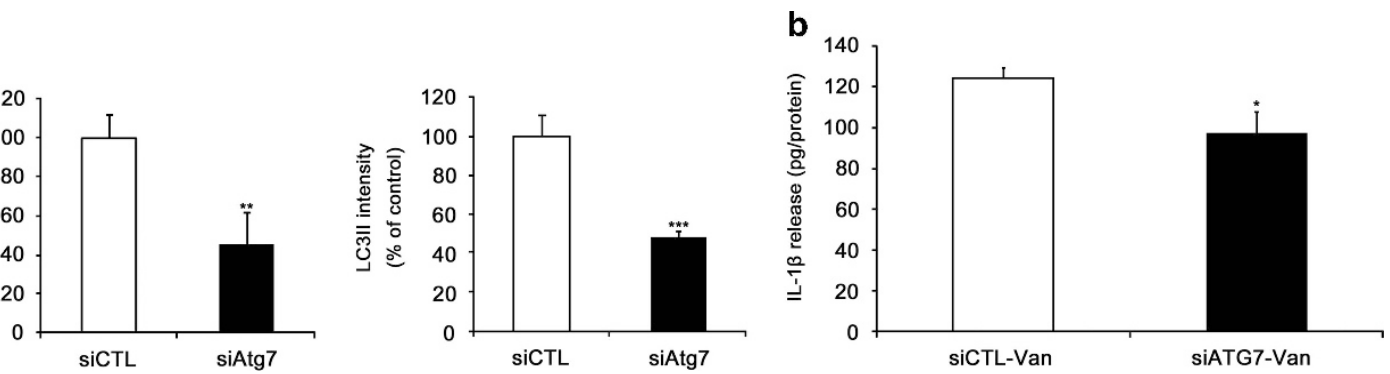

Figure 5 Decrease of IL-1 $\beta$ release by Atg7 knockdown. (a) Representative western blots of Raw264.7 cells transfected with siCTL (control) and siAtg7. siAtg7 effectively reduces the levels of Atg7 and LC3-II. (b) Representative graphs of enzyme-linked immunosorbent assay data showing IL-1 $\beta$ in vancomycin-treated Raw264.7 cells. IL-1 $\beta$ release is lower in siAtg7-transfected cells in comparison to siCTL-transfected cells. ${ }^{*} P<0.05$ vs $C T L$, ${ }^{* *} P<0.01$ vs CTL, ${ }^{* * *} P<0.005$ vs CTL. 
vancomycin is not a solution, but developing a new medicine could be the answer. This new medicine should demonstrate the same or better antibiotic effects compared with vancomycin, but should not demonstrate proinflammatory effects or inhibit autophagy.

\section{CONFLICT OF INTEREST}

The authors declare no conflict of interest.

\section{ACKNOWLEDGEMENTS}

This work was supported by Medical Research Center Program through the National Research Foundation of Korea (NRF) funded by the Ministry of Science, ICT \& Future Planning (2008-0062286). We thank Hyun-Soo Kim for assistance with the editing of this manuscript.

1 Klionsky, D. J. \& Emr, S. D. Autophagy as a regulated pathway of cellular degradation. Science 290, 1717-1721 (2000).
2 Levine, B., Mizushima, N. \& Virgin, H. W. Autophagy in immunity and inflammation. Nature 469, 323-335 (2011).

3 Levine, B. \& Deretic, V. Unveiling the roles of autophagy in innate and adaptive immunity. Nat. Rev. Immunol. 7, 767-777 (2007).

4 Deretic, V. Autophagy as an immune defense mechanism. Curr. Opin. Immunol. 18, 375-382 (2006).

5 Nakahira, K. et al. Autophagy proteins regulate innate immune responses by inhibiting the release of mitochondrial DNA mediated by the NALP3 inflammasome. Nat. Immunol. 12, 222-230 (2011).

6 Sadasivan, S. et al. Acute NMDA toxicity in cultured rat cerebellar granule neurons is accompanied by autophagy induction and late onset autophagic cell death phenotype. BMC Neurosci. 11, 21 (2010).

7 Park, J. et al. Haloperidol and clozapine block formation of autophagolysosomes in rat primary neurons. Neuroscience 209, 64-73 (2012).

8 Katayama, H., Yamamoto, A., Mizushima, N., Yoshimori, T. \& Miyawaki, A. GFP-like proteins stably accumulate in lysosomes. Cell Struct. Funct. 33, 1-12 (2008).

9 Al-Banna, N. A. et al. Impact of antibiotics on the microcirculation in local and systemic inflammation. Clin. Hemorheol. Microcirc. 53, 155-169 (2013).

10 Schnaith, A. et al. Staphylococcus aureus subvert autophagy for induction of caspaseindependent host cell death. J. Biol. Chem. 282, 2695-2706 (2007).

11 Hazlewood, K. A., Brouse, S. D., Pitcher, W. D. \& Hall, R. G. Vancomycin-associated nephrotoxicity: grave concern or death by character assassination? Am. J. Med. 123, 182 e181-182 e187 (2010). 